\title{
O PÓLO INFERIOR DO BAÇO DE RATOS E A OXIGENOTERAPIA HIPERBÁRICA
}

\author{
Isabel Cristina Andreatta lemos Paulo, Danilo Nagib Salomão Paulo*, Thiago Antunes Ferrari, Thiago Caetano Valadäo de Azeredo, Alcino lázaro da Silva
}

Trabalho realizado no Laboratório da Disciplina de Fundamentos da Cirurgia, Escola Superior de Ciências, Santa Casa de Misericórdia de Vitória, ES

\author{
*Correspondência \\ Rua Santa Rita de Cássia, 777 \\ Bairro de Lourdes - Vitória - ES \\ CEP 29042-753 \\ Tel: (27) 3323-4827/ \\ 9989-8290 \\ danilo.vix@zaz.com.br
}

\begin{abstract}
RESUMO
OBjetivo. Estudar aspectos funcionais e morfológicos do pólo inferior do baço de ratos tratados ou não com oxigênio hiperbárico no pós-operatório.

Métodos. Foram operados 79 ratos, Wistar, pesando 248,7g \pm 27 , distribuídos em dois grupos: $A$ - Simulação $(n=40)$, $B$ - pólo inferior ( $n=39$ ), e divididos em dois subgrupos: I I e 70 dias. Cada subgrupo foi subdividido em não tratado com oxigênio hiperbárico (nt) (AI Int-n=10, BII nt-n=13, A70nt-n=10, B70nt-n=9) e tratado (t); Al It-n=10, B| It- n=9, $A 70 t-n=10, B 70 t-n=8)$. Foram dosados os lípides e imunoglobulinas e contadas as plaquetas e os corpúsculos de HowellJolly no pré e no pós-operatório. O baço e o pólo inferior foram retirados para histologia.
\end{abstract}

Resultados. Houve aumento do colesterol total $(p=0,002)$, VLDL-colesterol e triglicérides $(p=0,003)$, da LDL-colesterol $(p=0,0 \mid 3)$ no subgrupo $B$ I Int e não houve alteração nos demais subgrupos. A IgM caiu nos subgrupos $B$ I It $(p=0,007)$, $B \mid \operatorname{lnt}(p=0,0000)$, $B 70$ nt $(p=0,0005)$ e $B 70 t(p=0,02)$ e não alterou no grupo simulação. $O$ número de plaquetas aumentou nos subgrupos $B \mid$ Int $(p=0,002), B \mid I t(p=0,01)$ e não alterou nos demais subgrupos. Os corpúsculos de HowellJolly foram menos numerosos no subgrupo $B 70$ nt que no $B \mid I n t(p=0,0 \mid 9)$. A viabilidade do pólo inferior foi melhor no subgrupo BI It que no B I Int; no subgrupo B70 que no BII e não diferiu entre os subgrupos B70t e B70nt.

ConcLusä̃o. A função e a viabilidade do pólo inferior remanescente melhoraram tardiamente. Os animais submetidos à oxigenoterapia hiperbárica apresentaram melhor viabilidade e função do pólo inferior precocemente, mas não tardiamente.

UnITERMOs: Esplenectomia/métodos. Baço/metabolismo. Efeitos adversos/esplenectomia.

\section{INTRODUÇÃo}

A esplenectomia total pode provocar complicações no transoperatório e no pós-operatório'. Dessas, a mais preocupante é a infecção fulminante que foi relatada por King e Schumaker $\mathrm{rr}^{2}$ e foi recentemente revista ${ }^{3}$. As complicações infecciosas, após a retirada do baço, foram também observadas em animais de experimentação $0^{4,5}$. As alterações no metabolismo lipídico têm sido relatadas após esplenectomia total tanto em seres humanos ${ }^{6}$ quanto em animais de experimentação $0^{7-11}$ e podem causar aterosclerose ${ }^{7}$. Por essas razões têm sido valorizadas as operações conservadoras sobre o baço que compreendem as oclusões vasculares ${ }^{12-16}$, esplenorrafias ${ }^{17}$, esplenectomias parciais ${ }^{18-20}$ e os auto-implantes ${ }^{21,22}$. Dessas, a mais recentemente descrita, em cães, foi a esplenectomia subtotal com preservação do pólo inferior, mesmo com a ligadura dos vasos esplênicos principais ${ }^{23}$. Nessa operação, observou-se aumento significante do colesterol e da fração LDL no pós-operatório mediato, e sugeriu-se a possibilidade de isquemia do pólo inferior remanescente. Por esse motivo, seria interessante utilizar um método para combater a isquemia e, possivelmente, melhorar a função do pólo inferior, no pós-operatório mediato.

A oxigenoterapia hiperbárica $(\mathrm{OHB})$ é um método terapêutico no qual o indivíduo é submetido à inalação intermitente de oxigênio $\left(\mathrm{O}_{2}\right)$ a 100\%, a uma pressão maior que a atmosférica, no interior de uma câmara hiperbárica ${ }^{24}$. Essa modalidade terapêutica tem sido utilizada no tratamento de várias doenças. No Brasil, a Resolução CFM I 457/95 do Conselho Federal de Medicina regulamentou seu uso nas seguintes indicações: embolia gasosa, embolia traumática pelo ar, envenenamento por monóxido de carbono, gangrena gasosa clostridiana, doença de Fournier, isquemias agudas traumáticas, queimaduras térmicas ou elétricas, lesões por irradiação, osteomielite, retalhos ou enxertos comprometidos ou de risco. No nível experimental demonstrou-se que a $\mathrm{OHB}$ tem efeito angiogênico ${ }^{25,26}$, estimula a formação de circulação colateral $^{27}$, aumenta o conteúdo arterial de oxigênio e a elasticidade dos glóbulos vermelhos ${ }^{28}$, acelera a cicatrização do baço após a ligadura simultânea da artéria e veia esplênicas ${ }^{29}$, além de atuar beneficamente em processos inflamatórios tais como: pancreatite ${ }^{30-32}$, colite ${ }^{33,34}$, hepatite crônica ${ }^{35}$ e aterosclerose ${ }^{36}$.

Considerando-se os efeitos da OHB, levantou-se a hipótese de que esse método poderia evitar a isquemia do pólo inferior e, possivelmente, melhorar a viabilidade e a função desse remanescente. Desse modo, poder-se-iam evitar ou atenuar as complicações decorrentes da hipofunção esplênica. Daí realizou-se esta pesquisa com o objetivo de estudar aspectos funcionais e morfológicos do pólo inferior do baço de ratos, tratados ou não com oxigênio hiperbárico, no pós-operatório.

\section{MÉTOdos}

Este estudo seguiu as recomendações do COBEA (Colégio Brasileiro de Experimentação Animal) e foi aprovado pelo Comitê de Ética 
PAULO ICAL ET AL.

em Pesquisa da Universidade Federal de Minas Gerais conforme documento de no ETIC 100/2006.

Foram operados 79 ratos machos, Wistar, pesando entre 192 g. e 29 I g. (M.A248,7 \pm 27 ), distribuídos aleatoriamente em dois grupos: A - Simulação $(n=40) ; B$ - pólo inferior $(n=39)$. Os grupos foram divididos em dois subgrupos: II dias e 70 dias. Cada subgrupo foi subdividido em não tratado com oxigênio hiperbárico (nt) $(A|| n t-n=10, B|| n t-n=13, A 70 n t-n=10, B 70 n t-n=9)$ e tratado (t); $A|| t-n=10, B|| t-n=9, A 70 t-n=10, B 70 t-n=8)$.

Após jejum de 12 horas, os ratos foram submetidos à analgesia com $25 \mathrm{mg} / \mathrm{Kg}$ de cloridrato de ketamina no músculo da pata posterior do animal, pesados (balança eletrônica Filizola ${ }^{\circledR}$-sensibilidade de Ig) e submetidos à punção cardíaca transtorácica para colheita de I,2 mL de sangue e dosagem dos lípides plasmáticos (Colesterol total, HDLcolesterol, LDL-colesterol, VLDL-colesterol e triglicérides), das imunoglobulinas, e contagem dos corpúsculos de Howell-Jolly e das plaquetas. Cada animal foi identificado com uma marca na orelha e devolvido à sua gaiola de origem. $\mathrm{O}$ sangue colhido $(\mathrm{I}, 2 \mathrm{~mL})$ foi dividido igualmente em um frasco com EDTA para realização de hemograma, leucograma e contagem de plaquetas, e em outro frasco, sem anticoagulante, para dosagem de imunoglobulinas e lípides. A dosagem das imunoglobulinas foi feita pelo método de turbidimetria, com o aparelho Behring ${ }^{\circledR}$ Turbitimer (Marburg-Alemanha), e a dos lípides no aparelho Dimension AR DADE BEHRING ${ }^{\circledR}$ (Illinois, EUA). A contagem das hemácias, plaquetas e leucócitos foi realizada em equipamento automatizado Cell-Dyn 3700 da Abott ${ }^{\circledR}$ (Illinois-USA). Para análise dos corpúsculos de Howell-Jolly em cada dez campos, foram feitas lâminas de esfregaço sanguíneo que foram analisadas em microscópio Olympus DX40 com objetiva de $100 \mathrm{mHz}$.

A anestesia dos ratos foi realizada com uma mistura de cloridrato de ketamina (50 mg/Kg) e cloridrato de xylasina (3 mg/Kg) aplicada por via intraperitoneal. Após anti-sepsia da parede abdominal com polivinilpirrolidona iodo tópico, foi realizada uma laparotomia mediana medioabdominal com $2 \mathrm{~cm}$. de comprimento, e exame da cavidade abdominal. Nos animais do grupo I, foi realizada a manipulação do baço. Nos animais do grupo 2 (pólo inferior), foi realizada a esplenectomia subtotal com preservação do pólo inferior, mesmo com a ligadura dos vasos esplênicos principais, conforme técnica já descrita (Figura I) ${ }^{23}$. A parede abdominal foi suturada em dois planos com mononáilon 4.0, chuleio simples.

A oxigenoterapia hiperbárica $(\mathrm{OHB})$ foi feita de acordo com o seguinte protocolo: a) imediatamente após a recuperação anestésica, os ratos foram colocados na câmara hiperbárica ${ }^{29}$, onde a pressão de oxigênio foi ajustada progressivamente durante 15 minutos até 2,5 atmosferas; b) o tempo de exposição à oxigenoterapia hiperbárica foi de 90 minutos; e a despressurização, de 15 minutos; c) nos três primeiros dias, foram feitas duas sessões de OHB diariamente e, nos sete dias seguintes, apenas uma sessão diária. Retirados da câmara, os animais foram devolvidos às suas gaiolas de origem onde receberam água e ração à vontade e paracetamol $200 \mathrm{mg} / \mathrm{Kg}$ via oral dissolvida na água do bebedouro. No $11^{\circ}$ dia de pós-operatório, em um subgrupo, e no $70^{\circ}$ dia em outro, foi realizada nova colheita de sangue para repetição dos exames laboratoriais. Após a colheita, os animais foram mortos com dose letal de pentobarbital sódico. A seguir foram pesados, e feita uma incisão
Figura I- Esplenectomia subtotal com preservação do pólo inferior, mesmo com a ligadura dos vasos esplênicos principais, em ratos ${ }^{14}$. Observar que as ligaduras dos ramos dos vasos esplênicos principais são feitas rentes ao baço (setas).

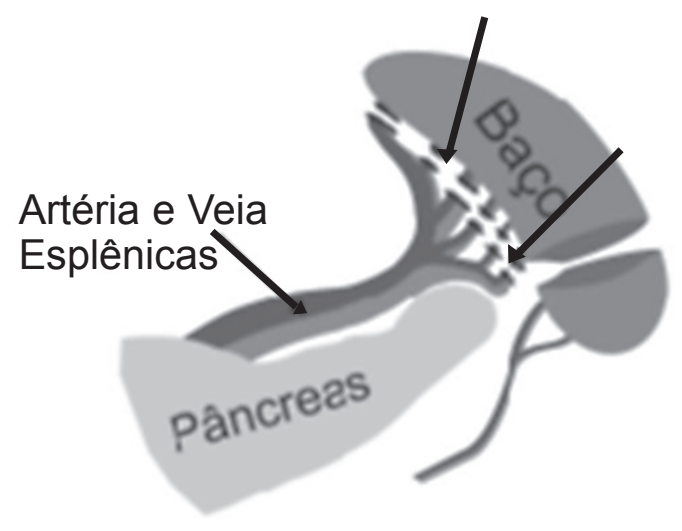

em $U$ invertido na parede abdominal e exame da cavidade. Foram examinadas as vísceras abdominais, o aspecto dos baços do grupo simulação e do pólo inferior. Para a avaliação histológica, o baço e o pólo inferior foram retirados, fixados em formol e incluídos em parafina e corados pela hematoxilina-eosina. A microscopia foi realizada por um anátomo-patologista em microscópio binocular. Os cortes foram analisados quanto às alterações morfológicas.

As análises estatísticas utilizadas foram: a) análise de regressão linear para calcular o percentual médio de massa do pólo inferior no grupo B. Isso foi feito dividindo-se o peso desse pólo pelo peso ideal do baço para cada animal do grupo simulação, multiplicando-se o resultado por 100. O cálculo do peso ideal do baço foi feito utilizando-se a fórmula: peso do baço $=1,80 \times$ peso corporal $+230,49$. Foi obtida a média do percentual da massa remanescente esplênica com o respectivo desviopadrão; b) Anova para amostras relacionadas para comparar os pesos do pré-operatório com os do pós-operatório num mesmo grupo; c) o teste $t$ de Student para amostras relacionadas a fim de comparar os valores do colesterol e frações e triglicérides, imunoglobulinas e plaquetas do pré-operatório com os do pós-operatório mediato e tardio; d) o teste do Qui-quadrado para comparar o número de animais sobreviventes e a freqüência dos corpúsculos de Howell Jolly entre os grupos. O teste exato de Fisher foi utilizado quando uma das freqüências esperadas foi inferior a 5. Os valores de $p$ foram considerados significantes, quando iguais ou menores que 0,05 ou $5 \%$.

\section{Resultados}

Não houve diferença significante de peso entre os dois grupos de animais no pré-operatório. Nos subgrupos Al I e B I I houve diminuição de peso independentemente de os animais terem sido submetidos ou não à oxigenoterapia hiperbárica. Essa queda foi significante no subgrupo $B$ I It $(p=0,03)$. Houve aumento de peso nos subgrupos $A 70 t$ $(p=0,0000)$, A70nt $(p=0,03), B 70 t(p=0,000 \mathrm{I})$ e B70nt $(p=0,00 \mathrm{I})$.

O percentual médio de massa do pólo inferior remanescente foi $38,87 \% \pm 6,05 \%$. 
O número de ratos sobreviventes no grupo simulação foi 100\% independentemente de terem sidos tratados ou não com oxigênio hiperbárico. No subgrupo II dias, não houve diferença significante entre a freqüência de sobreviventes do grupo simulação e do pólo inferior, tratados ou não com oxigênio hiperbárico. No subgrupo 70 dias, não houve também diferença significante entre a freqüência de sobreviventes do grupo do pólo inferior e do grupo simulação tratado e não tratado com oxigênio hiperbárico.

Nos subgrupos Al It e Al Int, os níveis de colesterol total e frações não apresentaram alterações significantes no $11^{\circ}$ dia de pósoperatório em relação ao pré-operatório. No subgrupo B I Int houve aumento do colesterol $(p=0,002)$, da LDL-colesterol $(p=0,0$ I3), VLDL-colesterol $(p=0,003)$ e triglicérides $(p=0,003)$ no pós-operatório em relação ao pré-operatório. A HDL-colesterol não alterou. Nos animais submetidos à oxigenoterapia hiperbárica não houve alterações nos níveis de colesterol e frações e triglicérides no $11^{\circ}$ dia de pós-operatório em relação ao pré-operatório. Nos subgrupos A70t, A70nt, B70t e B70nt não houve alterações dos lípides no $70^{\circ}$ dia de pós-operatório em relação ao pré-operatório (Tabela I).

As $\lg G$ e IgA não apresentaram alterações significantes nos subgrupos A I I, A70, B I I e B70 no pós-operatório em relação ao pré-operatório, nos animais tratados ou não com oxigênio hiperbárico. A IgM não apresentou alterações nos animais do grupo All e A70, tratados ou não com oxigênio hiperbárico. Essa imunoglobulina diminuiu nos subgrupos $B \mid$ It $(p=0,007), B$ I Int $(p=0,0000)$, B70nt $(p=0,0005)$ e B70t $(p=0,02)$. Os corpúsculos de Howell-Jolly estiveram ausentes no pré e pós-operatório dos subgrupos Al It, Al Int, A70t e A70nt e no pré-operatório dos subgrupos B I I e B70 tratados ou não com oxigênio hiperbárico. No pós-operatório do subgrupo Bl Int foram observados 34 corpúsculos em II ratos; no subgrupo B I It, dez corpúsculos em 7 ratos. No subgrupo B70nt foram observados cinco corpúsculos em oito ratos; e, no subgrupo B70t, um corpúsculo em sete ratos. O número de corpúsculos do subgrupo I Int foi maior que o número de corpúsculos do subgrupo 70 nt $(p=0,019)$ e não diferiu no subgrupo tratado.
Os níveis de plaquetas, no grupo $A$, não apresentaram alterações significantes nos subgrupos II dias e 70 dias, em relação ao préoperatório. Esse fato ocorreu não só nos animais tratados com oxigênio hiperbárico no pós-operatório, mas também nos animais não tratados. $\mathrm{O}$ aumento do número das plaquetas foi significante no subgrupo $\mathrm{B}$ I It $(p=0,0$ I), no subgrupo $B \mid$ Int $(p=0,002)$. No pós-operatório tardio não houve alterações do número de plaquetas no subgrupo B70, tratado ou não com oxigênio hiperbárico.

O aspecto macroscópico do baço foi normal no grupo simulação. O órgão apresentava poucas aderências à parede abdominal ou à vísceras adjacentes. Esse aspecto foi semelhante no grupo de animais tratados ou não com oxigênio hiperbárico no pós-operatório. O aspecto macroscópico dos pólos inferiores não diferiu entre os grupos tratados e não tratados com OHB. As aderências estiveram presentes em todos os casos, algumas vezes ao estômago, outras vezes às alças intestinais e outras à parede abdominal. Nos locais das aderências, principalmente ao omento, observaram-se vasos sanguíneos.

O aspecto microscópico do baço, nos animais do grupo simulação, tratados ou não com oxigênio hiperbárico, foi semelhante. Observouse hemossiderose, hematopoiese extramedular e presença de hemofagocitose na polpa vermelha. Nos animais do subgrupo BI Int, observou-se periesplenite fibrosa, necrose coagulativa extensa da polpa vermelha, áreas de calcificação distrófica da polpa vermelha e histiocitose, além de hemofagocitose. No subgrupo BI It observou-se bainha periarteriolar mais preservada e maior população linfóide. No subgrupo B70nt observou-se fibrose periesplênica, esplenose, hemossiderose, hematopoese extramedular, histiocitose e áreas de depleção linfocitária. No subgrupo B70t o aspecto foi semelhante.

\section{Discussão}

O pólo inferior remanescente da esplenectomia subtotal, mesmo com a ligadura dos vasos esplênicos principais, apresentou melhor função tardiamente. Esse fato ficou demonstrado quando se observou que não houve alteração significante do colesterol

\begin{tabular}{|c|c|c|c|c|c|c|c|c|c|c|}
\hline \multirow[t]{2}{*}{ Grupo } & \multicolumn{2}{|c|}{ Colesterol } & \multicolumn{2}{|c|}{$\mathrm{HDL}$} & \multicolumn{2}{|c|}{ LDL } & \multicolumn{2}{|c|}{ VLDL } & \multicolumn{2}{|c|}{ Triglicérides } \\
\hline & Pré & Pós & Pré & Pós & Pré & Pós & Pré & Pós & Pré & Pós \\
\hline Allnt & $53,1 \pm 6,5$ & $52,7 \pm 6,5$ & $21,5 \pm 2,7$ & $21,7 \pm 2,6$ & $22,4 \pm 2,7$ & $21,9 \pm 2,8$ & $9,0 \pm 1,5$ & $9,0 \pm 1,2$ & $45,8 \pm 7,1$ & $45,3 \pm 6,3$ \\
\hline Al lt & $55,6 \pm 6,3$ & $55,4 \pm 6,8$ & $22,5 \pm 2,3$ & $22,6 \pm 2,4$ & $23,7 \pm 2,6$ & $23,3 \pm 3,0$ & $9,3 \pm 1,4$ & $9,4 \pm 1,4$ & $46,7 \pm 7,3$ & $47,0 \pm 7,3$ \\
\hline A70nt & $53,2 \pm 6,1$ & $53,1 \pm 6,2$ & $21,8 \pm 2,6$ & $21,8 \pm 2,5$ & $21,7 \pm 3,0$ & $21,5 \pm 3,1$ & $9,6 \pm 1,4$ & $9,7 \pm 1,1$ & $48,4 \pm 7,0$ & $48,9 \pm 5,8$ \\
\hline A70t & $55,8 \pm 5,9$ & $55,2 \pm 5,4$ & $22,7 \pm 2,2$ & $22,6 \pm 2,1$ & $23,2 \pm 2,7$ & $22,8 \pm 2,6$ & $9,7 \pm 1,1$ & $9,6 \pm 1,0$ & $48,6 \pm 5,9$ & $48,4 \pm 5,0$ \\
\hline Bllnt & $54,4 \pm 3,6$ & $55,8 \pm 3,3^{* * *}$ & $22,2 \pm 1,4$ & $22,2 \pm 1,5$ & $22,5 \pm 1,7$ & $23,7 \pm 1,5 *$ & $9,6 \pm 0,8$ & $9,8 \pm 0,9 * *$ & $48,2 \pm 4,1$ & $49,3 \pm 4,6$ *** \\
\hline Bllt & $56,0 \pm 11,0$ & $55,7 \pm 10,3$ & $23,2 \pm 4,1$ & $23,2 \pm 4,3$ & $23,2 \pm 4,9$ & $22,9 \pm 4,1$ & $9,6 \pm 2,1$ & $9,5 \pm 1,9$ & $48,0 \pm 10,5$ & $47,8 \pm 9,9$ \\
\hline B70nt & $55,3 \pm 3,8$ & $55,6 \pm 4,2$ & $22,6 \pm 1,3$ & $22,7 \pm 1,6$ & $23,0 \pm 1,8$ & $23,1 \pm 2,0$ & $9,6 \pm 0,9$ & $9,8 \pm 0,8$ & $48,3 \pm 4,9$ & $49,1 \pm 4,2$ \\
\hline B70t & $53,4 \pm 9,8$ & $53,1 \pm 9,5$ & $22,3 \pm 3,8$ & $22,1 \pm 3,7$ & $22,1 \pm 4,2$ & $21,9 \pm 4,1$ & $8,9 \pm 1,8$ & $9,0 \pm 1,8$ & $44,4 \pm 9,1$ & $45,1 \pm 9,2$ \\
\hline
\end{tabular}

Grupos: A - simulação; B - pólo inferior; I I - término do experimento no I I dia de pós-operatório; 70 - término do experimento no $70^{\circ}$ dia de pós-operatório; nt - não tratado com oxigênio hiperbárico; $t$ - tratado. * $\mathrm{p}<0,05$ - do pré-operatório (Pré) para o pós-operatório da LDL-colesterol no subgrupo BI Int. *** $p<0.01$-do pré-operatório para o pós-operatório do Colesterol total, VLDL-colesterol e triglicérides no subgrupo BI Int. 
total, LDL-colesterol, VLDL-colesterol e triglicérides, no $70^{\circ}$ dia de pós-operatório, em relação ao pré-operatório, e houve aumento significante desses lípides no $11^{\circ}$ dia. Além disso, o número de corpúsculos de Howell-jolly foi menor no subgrupo B70nt que no $\mathrm{B} \mid \mathrm{Int}(\mathrm{p}=0,0 \mid 9)$, e as plaquetas que aumentaram no $1 \mathrm{I}^{\circ}$ dia de pós-operatório nos subgrupos $B \mid \operatorname{lnt}(p=0,002)$ e $B \mid$ It $(p=0,0 \mid)$ não sofreram alterações no $70^{\circ}$ dia em relação ao pré-operatório. A queda da IgM foi mais significante no subgrupo I Int que no subgrupo 70 nt $(p=0,0000$ versus $p=0,007)$. Essa melhora de função decorre provavelmente da melhora da viabilidade esplênica, conforme ficou demonstrado neste estudo e em estudo anterior$^{8}$. Foi sugerido que o pólo inferior, no pós-operatório imediato, pode apresentar isquemia ${ }^{23}$. De fato, a análise microscópica neste estudo mostra sinal sugestivo de isquemia, tal como área de necrose coagulativa. É importante lembrar que a isquemia é causa de aderências ${ }^{37}$, e que o omento freqüentemente se encontra aderente a órgãos isquêmicos. Sabe-se que o omento apresenta grande potencial de neovascularização por meio de angiogênese. Os adipócitos e as células endoteliais dos vasos sanguíneos do omento estariam envolvidos nesse process ${ }^{38}$. A capacidade angiogênica do omento maior foi também demonstrada em transplante hepático humano que apresentou vascularização espontânea nas aderências teciduais ${ }^{39}$. Conforme relatamos, freqüentemente observamos o omento aderente ao pólo inferior e, nesses locais, vasos sangüíneos. Por isso, o estudo da angiogênese desse remanescente seria interessante.

Os animais submetidos à oxigenoterapia hiperbárica apresentaram melhor viabilidade e função do pólo inferior precocemente, mas não tardiamente. De fato, os níveis de lípides do subgrupo I Int aumentaram significantemente no pós-operatório, e os níveis do subgrupo IIt não alteraram. Além disso, a IgM caiu mais significantemente, e as plaquetas aumentaram mais significantemente no subgrupo II nt, em relação ao subgrupo I It. Por outro lado, tardiamente, não houve diferença na função do subgrupo B70t e B70nt. Cumpre ressaltar que, em coelhos, a OHB reduziu a progressão e acelerou a regressão da aterosclerose por mecanismo antioxidante ${ }^{23} \mathrm{e}$ que o LDL oxidado está implicado na gênese da referida doença. Desse modo é possível que a OHB interfira nos níveis de LDL direta ou indiretamente. De maneira indireta, a OHB melhoraria a viabilidade do pólo inferior e conseqüentemente a função.

A melhor viabilidade do pólo inferior dos animais do subgrupo I I tratados com oxigênio hiperbárico é observada na microscopia onde a bainha arteriolar é mais preservada e há maior população linfóide em relação ao subgrupo IInt. Considerando que a OHB tem efeito benéfico no processo inflamatório ${ }^{30-36}$, que ela estimula a circulação colatera ${ }^{27}$ e tem efeito angiogênico ${ }^{25,26}$, é admissível que essa modalidade de tratamento tenha contribuído para melhorar a viabilidade do pólo inferior. A oxigenoterapia hiperbárica tardiamente não interferiu na viabilidade e na função conforme pudemos observar na análise dos resultados funcionais e morfológicos. Cumpre lembrar que a $\mathrm{OHB}$ foi aplicada apenas nos dez primeiros dias do pós-operatório, e a avaliação foi realizada 60 dias após. No $70^{\circ}$ dia do pós-operatório, independentemente da oxigenoterapia hiperbárica, o pólo inferior apresentou melhor função e viabilidade que no $11^{\circ}$ dia. Estudos sobre a evolução do pólo inferior, em animais não tratados com oxigênio hiperbárico, estão em andamento e os resultados iniciais (dados não publicados) sugerem que o pólo inferior cresce após um determinado período, conforme foi sugerido ${ }^{40}$. É possível, pois, que com esse crescimento ocorra melhora da função. Van Wick et al., ${ }^{41}$, em trabalho experimental em ratos, consideram necessária a manutenção de um terço do tecido esplênico total para restaurar a função esplênica. Nesse estudo, conforme se relatou, o percentual médio do pólo inferior remanescente foi de $38,87 \%$. Mesmo que no pós-operatório mediato tenha ocorrido isquemia desse remanescente, no pós-operatório tardio, com o crescimento desse pólo, vai haver recuperação funcional, conforme foi observado ${ }^{23}$.

\section{Conclusão}

Do que foi exposto pode-se concluir: a função e a viabilidade do pólo inferior melhoram tardiamente. Os animais submetidos à oxigenoterapia hiperbárica apresentaram melhor viabilidade e função do pólo inferior precocemente, mas não tardiamente.

\section{Agradecimentos}

Os autores agradecem ao Instituto Solidário do Espírito Santo pelo auxílio financeiro.

\section{Conflito de interesse: não há}

\section{SUMMARY}

THE SPLENIC INFERIOR POLE OF RATS AND HYPERBARIC OXYGEN

OBIECTIVE. TO study the functional and morphological features of the lower pole of the spleen in rats submitted, or not, to postoperative hyperbaricoxygen therapy.

Methods. Seventy-nine Wistar rats, weighing $248.7 \pm 27$ g, divided into two groups [group $A$ - simulation $(n=40)$, group $B$ - lower pole $(n=39)]$ underwentsurgery and were subdivided into two groups: I I and 70 postoperative days. Each subgroup was subdivided into animals not treated (nt) (Al Int, $n=10 ; B \mid$ Int, $n=13 ; A 70 n t, n=10 ; B 70 n t, n=9$ ) and treated with hyperbaric oxygen ( $t$ ) (AI It, $n=10 ; B \mid I t, n=9 ; A 70$, $n=10 ; B 70 t, n=8)$. Blood was collected for measurement of lipids and immunoglobulins, platelet and Howell-Jolly body count before and after surgery. The spleen and lower pole were removed for histology.

RESULTS. There was an increase of total cholesterol $(p=0.002), V L D L-$ cholesterol and triglycerides $(p=0.003)$ and of $L D L$-cholesterol $(p=0.013)$ in subgroup $B / I n t$, and no alterations were observed in the other subgroups. IgM decreased in subgroups $B$ I It $(p=0.007), B$ I Int $(p=0.0000), B 70 n t(p=0.0005), B 70 t(p=0.02)$, and no change was observed in the simulation group. The number of platelets increased in subgroups $B /$ Int $(p=0.002)$ and $B / I t(p=0.01)$ and remained unchanged in the other subgroups. Howell-Jolly bodies wereless numerous in subgroup B70nt than in subgroup $B$ I Int $(p=0.019)$. Lower pole viability was better in subgroup $B I$ It than in $B I$ Int and in subgroup $B 70$ than in $B / I$, and did not differ between subgroups B70t and B70nt.

CONCLUSION. Function and viability of the remaining lower pole improved during the late postoperative period. Viability and function of the 
lower pole were better during the early but not during the late postoperative period, in animals submitted to hyperbaric oxygen therapy. [Rev Assoc Med Bras 2008; 54(I): 77-8I]

KEY WORDS: Splenectomy/methods. Spleen/metabolism. Adverse effects/splenectomy.

\section{REFERÊNCIAS}

I. Petrovic M, Popovic M, Knezevic S, Matic S, Gotic M, Milovanovic A, et al. Intraoperative and postoperative complications of splenectomy. Acta Chir lugosl. 2002;49:81-4.

2. King H, Shumacker Jr HB. Splenic studies. Ann Surg. 1952; I 36:239-42.

3. Marques RG, Petroianu A. Overwhelming postsplenectomy infection. Arq Gastroenterol. 2003:40:47-54.

4. Morris DH, Bullock FD. The importance of the spleen in resistance to infection. Ann Surg. 1919;70:513-21.

5. Andersson R, Alwmark A, Bengmark S. Outcome of pneumococcal challenge in rats after splenic artery ligation or splenectomy. Acta Chir Scand. 1986; 152:15-7.

6. Aviram M, Brook JG, Tatarsky, Levy Y, Carter A. Increased low-density lipoprotein levels after splenectomy: a role for the spleen in cholesterol metabolism in myeloproliferative disorders. Am J Med Sci. 1986;291:25-8.

7. Asai K, Kuzuya M, Naito M, Funaki C, Kuzuya F. Effects of splenectomy on serum lipids and experimental atherosclerosis. Angiology. 1988;39:497-504.

8. Paulo DNS, Lázaro da Silva A. Lipídios plasmáticos após esplenectomia total e parcial em cães. Rev Col Bras Cir. 200 I;28:264-70.

9. Jamel MJ, Refineti RA, Silva MM, Maciel ACC, Marelli GB. Papel do remanescente esplênico no perfil lipídico. Arq Bras Cir Dig. 2002; 1 5: 105-7.

10. Paulo ICAL, Paulo DNS, Kalil M, Guerra AJ, Guerzet EA, Silva AL. Effects of two types of diet on plasma lipids in rats submitted to splenic surgery. Rev Assoc Med Bras. 2007;53: 171-7.

I I. Petroianu A, Veloso DFM, Costa GR, Alberi LR. Effects of splenic surgeries on lipidograms of rats. Rev Assoc Med Bras. 2006;52:56-9.

12. Warshaw AL. Conservation of the spleen with distal pancreatectomy. Arch Surg. 1988; 123:550-3.

13. Zilberstein B, Sallet JA, Ramos A, Eshkenazy R. Vídeo laparoscopy for the treatment of bleeding esophageal varices. Surg Laparosc Endosc. | 997;7: | 85-9|.

14. Poulin EC, Mamazza J, Schlachta CM. Splenic artery embolization before laparoscopic splenectomy. An update. Surg Endosc. 1998; 12:870-5.

15. Schertler T, Pfammatter T, Eid K, Wildermuth S. Embolization of the splenic artery after splenic gunshot wound. Swiss Surg. 2003;9:87-91 .

16. Mignon F, Brouzes S, Breitel DL, Bastie JN, Poirier H, Legendre C, et al. Embolisation préopératoire selective permettant une splénectomie partielle por hamartome splénique. Ann Chir. 2003; 128: I 12-6.

17. Feliciano DV, Bitondo CG, Mattox KL, Rumisek JD, Burch JM, Jordan GL Jr. A four-year experience with splenectomy versus splenorrhaphy. Ann Surg. 1985;201:568-75.

I 8- Campos-Christo M. O estado da arte da cirurgia do baço, no início do século XXI. Rev Col Bras Cir. 2005;32:279-82.

19. Petroianu A. Esplenectomia subtotal e anastomose esplenorrenal proximal para o tratamento da hipertensão portal. Rev Bras Cir. 1983;73(2): 10 |-4.

20. Paulo DNS, Lázaro da Silva A, Cintra LC, Bof AM, Santiago DC, Ribeiro GB. Esplenectomia subtotal, em cães, com preservação do pólo inferior suprido por vasos do ligamento gastroesplênico. Rev Col Bras Cir. 1999:26: 147-52.

21. Holdsworth RJ. Regeneration of the spleen and splenic autotransplantation . Br J Surg. 1991;78:270-8.
22. Marques RG. Autoimplante esplênico em ratos: regeneração morfológica e função fagocitária [tese]. Belo Horizonte: Faculdade de Medicina, Universidade Federal de Minas Gerais; 2001.

23. Paulo DNS, Paulo ICAL, Kalil M, Vargas PM, Lázaro da Silva A, Baptista JFA, et al. Subtotal splenectomy preserving the lower pole in rats: technical, morphological and functional aspects. Acta Cir Bras. 2006;21:321-7.

24. Brettscneider L, Daloze PM, Huguet C, Porter KA, Groth CG, Hutchison $\mathrm{DE}$, et al. The use of combined preservation techniques for extended storage of orthotopic liver homografts. Surg Gynecol Obstet. 1968; 126:263-6.

25. Cundall JD, Gardiner A, Chin K, Laden G, Grout P, Duthie G. Hyperbaric oxygen in the treatment fecal incontinence secondary to pudendal neuropathy. Dis Colon Rectum. 2003;46: I 549-54.

26. Muhonen A, Haaparanta M, Grönroos T, Bergman J, Knuuti J, Hinkka S, et al. Osteoblastic activity and neoangiogenesis in distracted bone of irradiated rabbit mandible with or without hyperbaric oxygen treatment. Int J Oral Maxillofac Surg. 2004;33: 173-8.

27. Burt JT, Kapp JP, Smith RR. Hyperbaric oxygen and cerebral infarction in the gerbil. Surg Neurol. 1987;28:265-8.

28. Tolentino EC, Castro e Silva O, Zucoloto S, Souza ME, Gomes MC, Sankarankutty AK, et al. Effect of hyperbaric oxygen on liver regeneration in a rat model. Transplant Proc. 2006;38: 1947-52.

29. Paulo DNS, Kalil M, Grillo Junior LSP, Borges EB, Cintra LC, Pereira FEL, Lázaro da Silva A. Viability of the spleen in rats after ligation of the splenic vessels: effects of hyperbaryc oxygen therapy. Rev Assoc Med Bras. 2005;51:46-50.

30. Mas N, Isik AT, Mas MR, Comert B, Tasci I, Deveci S, et al. Hyperbaric oxygen-induced changes in bacterial translocation and acinar ultrastructure in rat acute necrotizing pancreatitis. J Gastroenterol. 2005;40:980-6.

31. Yasar M, Yildiz S, Mas R, Dundar K, Yildirim A, Korkmaz A, et al. The effect of hyperbaric oxygen treatment on oxidative stress in experimental acute necrotizing pancreatitis. Physiol Res. 2003;52: I I I-6.

32. Cheng HM, Shyr MH, Ueng SW, Chen MF. Hyperbaric oxygen therapy attenuates pancreatic microcirculatory derangement and lung edema in an acute experimental pancreatitis model in rats. Pancreas. 1998; 17:44-9.

33. Akin ML, Gulluoglu BM, Uluutku H, Erenoglu C, Elbuken E, Yildirim S, et al. Hyperbaric Oxygen improves healing in experimental rat colitis. Undersea Hyperb Med. 2002;29:279-85.

34. Rachmilewitz D, Karmeli F, Okon E, Rubenstein I, Better OS. Hyperbaric oxygen: a novel modality to ameliorate experimental colitis. Gut. 1998:43:512-8.

35. Liu W, Zhao W, Lu X, Zheng X, Luo C. Clinical pathological study of treatment of chronic hepatitis with hyperbaric oxygenation. Clin Med J. 2002; I I : I I 53-7.

36. Kudchodkar BJ, Wilson J, Lacko A, Dory L. Hyperbaric Oxygen reduces the progression and accelerates the regression of atherosclerosis in rabbits. Arterioscler Thromb Vasc Biol. 2000;20: I 637-43.

37. Ellis $\mathrm{H}$. The aetiology of post-operative abdominal adhesions. An experimental study. Br J Surg. 1962;50: 10-6.

38. Goldsmith HS, Griffith AL, Kupferman A, Catsimpoolas N. Lipid angiogenic factor from omentum. J Am Med Assoc. 1984;252:2034-46.

39. Silva PC, Jamel N, Refinetti RA, Manso JEF, Schanaider A. Development of blood vessels of the greater omentum in the hepatic lobe after vascular ligation. An experimental model in rats. Acta Cir Bras. 2006;21:416-20.

40. Torres OJM, Macedo EL, Picciani ERG, Nunes PMS, Costa JVG, Carvalho $\mathrm{BA}$, et al. Estudo histológico da regeneração esplênica de ratos submetidos a esplenectomia subtotal. Acta Cir Bras. 2000; 15: I - 12.

4I. Van Wick DB, White MH, White CL. Critical splenic mass for survival from experimental pneumococcemia. J Surg Res. 1980;28:14-7. 